\title{
¿Qué SABEMOS DE LA RAREZA EN ESPECIES VEGETALES? UN ENFOQUE GENÉTICO-DEMOGRÁFICO
}

\author{
Ligia Guadalupe Esparza-Olguín \\ Departamento de Ecología y Recursos Naturales, Facultad de Ciencias, Universidad Nacional Autónoma de México. \\ Ciudad Universitaria, México, 04510, México D.F. Correo-e: esparza_ligia@yahoo.com.mx
}

\begin{abstract}
Resumen: En este trabajo se presenta una recopilación de una serie de estudios sobre demografía y genética en especies vegetales raras. El propósito es analizar si existen patrones con respecto a la demografía y genética de poblaciones en especies raras. Del análisis, se obtuvo que en la mayoría de las especies estudiadas desde el punto de vista demográfico, se presentan tasas de crecimiento poblacional cercanas a la unidad, limitaciones importantes en la reproducción, bajos porcentajes de germinación, bajas tasas de reclutamiento y, en algunos casos, ciertos aspectos del proceso reproductivo asociados a eventos intermitentes. Con respecto a los aspectos genéticos, queda de manifiesto la existencia de una amplia gama de valores en la variabilidad genética de las especies raras. Así, es claro que las especies vegetales raras constituyen un ensamble heterogéneo. Por el momento, no es posible afirmar que existan patrones demográficos o genéticos generales en este grupo de especies.
\end{abstract}

Palabras clave: conservación, demografía, genética, rareza.

\begin{abstract}
In this paper I present a review of a number of studies that have addressed demographic or genetic aspects of rare plant species, with the purpose of analyzing whether there are any demographic or genetic patterns which characterize them. From this review, I observed that in the majority of the rare plant species studied from a demographic point of view, several common features appear: population growth rates close to unity, important reproductive limitations, low germinability, low recruitment rates, and in some cases, certain aspects of the reproductive process are associated to intermittent events. Regarding the genetic studies, the existence of a wide range of values in the genetic variability of rare species became clear. Therefore, it may be concluded that rare plant species constitute a biologically heterogeneous assemblage. At present it is not possible to conclude about the existence of general demographic or genetic patterns within this group.
\end{abstract}

Key words: conservation, demography, genetics, rarity.

$\mathbf{E}_{\mathrm{t}, \mathrm{s}}^{1}$ l estudio de la rareza como fenómeno biológico constituye un área de gran interés en el ámbito ecológico. Históricamente el concepto de rareza, desde el punto de vista biológico, ha sido utilizado como sinónimo de una abundancia baja y de un área de distribución restringida. Así, la mayoría de las definiciones se han basado principalmente en características como la abundancia, la distribución y la restricción del hábitat de las especies (Griggs, 1940; Drury, 1974; Harper, 1981; Rabinowitz, 1981; Cody, 1986; Soulé, 1986; Rabinowitz et al., 1986; Gaston, 1994; Kunin y Gaston, 1997). De los múltiples intentos por definir el término de rareza, el más conocido y empleado actualmente para el caso de especies vegetales es el propuesto por Rabinowitz (1981, Rabinowitz et al., 1986), quien reconoce que la rareza de las especies se da a diferentes niveles y puede ser de diferentes tipos. Esta autora reconoce básicamente tres tipos de rareza: 1) rareza biogeográfica (relacionada con la distribución de las especies), 2) rareza de hábitat (relacionada con el grado de restricción de hábitat de las especies), y 3) rareza demográfica (relacionada con la abundancia de las especies). A partir de este reconocimiento, distingue siete formas de rareza que dependen de diferencias dicotómicas en los tres criterios de rareza propuestos (área de distribución amplia o restringida, especificidad de hábitat extenso o limitado, y abundancia local alta o baja).

Dado que las especies raras tienen una mayor probabilidad de desaparecer que las especies comunes, el tema de la rareza se ha enmarcado preferentemente dentro de la biología de la conservación siendo, en cambio, muy pocos los estudios que abordan las causas y conse- 
cuencias ecológicas y evolutivas de la rareza (Rabinowitz, 1981; Gaston, 1994). El hecho de que el estudio de la rareza se haya enmarcado preferentemente dentro de la biología de la conservación ha aportado importantes fundamentos teóricos y prácticos para la instrumentación de programas de conservación, puesto que la mayoría de las especies amenazadas o en peligro de extinción son también especies raras (Primack, 1993; Gaston, 1994; Schemske et al., 1994; Hunter, 1996). Sin embargo, no se debe perder de vista que el estudio de la rareza es importante per se al igual que lo es el estudio de la competencia, el mutualismo, la estructura de las comunidades y otros temas de relevancia ecológica, pues nos llevan a entender los patrones de abundancia y distribución de las especies en la naturaleza. Por esta razón, es necesario realizar estudios en los que se intente reconocer las causas y consecuencias tanto ecológicas como evolutivas de la rareza.

Para reconocer las causas de la rareza, se vuelve crucial la determinación de los factores que ocasionan que una especie tenga una abundancia baja o una distribución restringida, tomando en cuenta que: 1) en ausencia de cualquier otro factor (i.e. antropogénico) la abundancia y la distribución de una especie están limitadas por variables ambientales (abióticas y bióticas), y 2) las poblaciones de especies raras están influenciadas por una gran cantidad de factores determinísticos y estocásticos (i.e. eventos naturales de disturbio, cambios azarosos en las tasas de natalidad y mortalidad, cambios en la calidad del hábitat, depresión endogámica, deriva génica, etc.), por lo que es muy probable que la rareza sea el resultado de la combinación de varios factores y no de uno solo (Kruckeberg y Rabinowitz, 1985; Soulé, 1986; Begon et al., 1990; Prober y Austin, 1990; Gaston, 1994; Root, 1998). ¿Cuánto sabemos acerca del comportamiento demográfico o de la estructura genética de las especies raras?, ¿es factible hablar de patrones demográficos o genéticos que caractericen a las especies raras? Si es así ¿cómo son estos patrones? En esta revisión pretendo analizar la información disponible hasta el momento con respecto a la demografía y la genética de poblaciones de especies raras con el propósito de contestar estas preguntas. A diferencia de la revisión publicada por Schemske et al. (1994) hace una década, en la que los autores evalúan los diferentes enfoques que se han utilizado para el estudio de especies raras con un énfasis en la conservación, en el presente trabajo pretendo brindar un panorama general sobre lo que se sabe de la rareza como fenómeno biológico, en particular con respecto a las características genéticas y demográficas de las especies raras en comparación con las que presentan especies comunes cercanamente emparentadas.

El fenómeno de la rareza en especies vegetales. Para abordar el análisis de los procesos que provocan que una especie sea rara, debe entenderse el fenómeno de la rareza, para lo cual se requiere definir claramente el concepto de rareza. De hecho, los primeros trabajos que abordaron este tema se enfocaron a definirla. Como se mencionó antes, las definiciones se han basado principalmente en la abundancia, la distribución y la restricción de hábitat de las especies (Griggs, 1940; Drury, 1974; Harper, 1981; Rabinowitz, 1981). Posteriormente comenzaron a generarse listados de especies raras para diferentes regiones, en gran medida con la finalidad de generar estrategias de conservación para estas especies (Cody, 1986; Hubbell y Foster, 1986; Rabinowitz et al., 1986; Andersen, 1989; Lahti et al., 1991; Pate y Hopper, 1994; Woolley y Kirkpatrick, 1999). Estos listados se han basado en el conocimiento de la distribución, la abundancia y la restricción del hábitat de diferentes taxa y se han convertido en un intento por encontrar patrones y comportamientos asociados a las especies raras.

Algunos autores sugieren que tanto la diversidad como la incidencia de especies raras dependen tanto de las características del hábitat, como de las características propias de cada taxón (Cody, 1986). Otros autores proponen que las especies que pertenecen a distintas formas de rareza presentan diferentes características biológicas, tales como su nivel de plasticidad, tolerancia a cambios ambientales y formas de dispersión específicas, entre otras (Rabinowitz et al., 1986). En otros estudios se señala que, tanto los disturbios naturales como los antropogénicos, pueden producir un efecto más dramático, en términos de extinciones locales y cambios en las tasas de reclutamiento y crecimiento, en las poblaciones de especies raras que en las poblaciones de especies comunes (Hubbell y Foster, 1986; Andersen, 1989).

En los ejemplos del párrafo anterior, los autores señalan la falta de información detallada sobre la biología de las especies raras, coincidiendo en que es indispensable profundizar en el tema para la formación de criterios adecuados de conservación y manejo de las especies raras, y para la comprensión del fenómeno de la rareza en sí mismo. La falta de información detallada que nos permita identificar características generales que pudieran estar determinando que unas especies sean raras y otras relativamente más comunes, ha generado un interés por desarrollar estudios en los que se aborde el análisis ecológico de estas especies, particularmente en el área de ecología de poblaciones, de manera complementaria al análisis de sus características genéticas. La finalidad de estos trabajos (e.g. Loveless y Hamrick, 1984; Falk y Holsinger, 1991; Bevill y Louda, 1999) no es sólo la de identificar aquellos atributos que puedan estar limitando la abundancia y la distribución de estas especies, sino también la de generar, como se mencionó con anterioridad, mejores propuestas para asegurar su persistencia.

La relevancia de contar con estudios genéticos para especies vegetales raras es clara, pues se sabe que la estructura y la variabilidad genética de las poblaciones juegan un 
papel muy importante en la distribución y abundancia de las especies vegetales (Milligan et al., 1994). Además, el conocimiento de la variabilidad genética en las poblaciones naturales y el análisis de la manera en la que ésta puede verse amenazada cuando las poblaciones están aisladas o cuando presentan un número reducido de individuos (que frecuentemente es el caso de las poblaciones de especies raras) es de suma importancia para la generación de propuestas de conservación y manejo, pues es esta variabilidad la que le brinda a las especies la posibilidad de adaptarse a las condiciones ambientales presentes y futuras (Holsinger y Gottilieb, 1991; Rosseto et al., 1995).

En relación con los estudios llevados a cabo con especies vegetales raras, puede constatarse que los de genética de poblaciones son más numerosos que los realizados en otros ámbitos de la biología, lo que ha permitido detectar algunas características generales de las especies raras a este nivel. Por ejemplo, se sabe que algunas de las consecuencias evolutivas más importantes de tener poblaciones pequeñas o aisladas (o pequeñas y aisladas a la vez) son la pérdida de variación genética por deriva génica, la acumulación de mutaciones deletéreas, la reducción del número de heterócigos asociada a procesos de endogamia y la depresión endogámica (Lande, 1988, 1995; Milligan et al., 1994; O’Brien, 1994; Ouborg et al., 1995; Lynch y Vaillancourt, 1995; Fischer y Matthies, 1998). A corto plazo, todo esto trae como consecuencia la reducción en la adecuación de los individuos y, a largo plazo, la reducción en la capacidad de adaptación de las poblaciones a cambios en el ambiente (Lande, 1988; Oostermeijer et al., 1994; Rossetto et al., 1995; Fischer y Matthies, 1998). Esto reafirma la idea de que las características genéticas de las poblaciones tienen influencia directa en su abundancia y su distribución.

La ecología de poblaciones en el análisis de la rareza. Los estudios que abordan aspectos de la ecología de poblaciones y la demografía de especies raras pueden dividirse en dos grandes grupos, según el enfoque que utilizan: 1) estudios comparativos en los cuales se contrastan las características de especies raras y especies comunes (Rabinowitz et al., 1989; Lahti et al., 1991; Prober, 1992; Kunin y Gaston, 1993; Gaston, 1994; Byers y Meagher, 1997; Walck et al., 1999), o de especies cercanamente emparentadas o ecológicamente similares que varían en su nivel de rareza (Fiedler, 1987; Louda, 1994; Baskin et al., 1997; Coates y Hamley, 1999), y 2) estudios uni-específicos que brindan información más detallada acerca de la biología de una especie rara en particular, con la finalidad de detectar los factores que limitan su abundancia y su distribución (Menges, 1990; Prober y Austin, 1990; Shmalzel et al., 1995; García, 1997; Fisher y Matthies, 1998; Wong y Sun, 1999).

En general, en los estudios comparativos realizados hasta la fecha, se aborda el papel que juegan aspectos como la competencia y las características del hábitat en el fenómeno de la rareza (e.g. Rabinowitz, 1981; Lahti et al., 1991; Prober, 1992; Walck et al., 1999). El común denominador que se enfatiza en los trabajos comparativos que contrastan características de especies raras y comunes, o de especies con diferentes niveles de rareza, es la "preferencia" de las especies raras por ambientes extremos, ya sea por los altos niveles de sequía, de disturbio (i.e. fuego) o de temperaturas extremas, así como la restricción de su hábitat (Prober, 1992; Coates y Hamley, 1999; Walck et al., 1999). Otra característica común en las especies de plantas raras pareciera ser su baja competitividad con respecto a especies vegetales comunes cercanamente emparentadas (Prober, 1992; Walck et al., 1999). Sin embargo, son tan pocos los trabajos comparativos y detallados realizados sobre este tema, que no es posible hacer generalizaciones al respecto. Claramente hace falta más información para poder decidir si los ejemplos antes mencionados constituyen patrones generales en especies raras.

En cuanto a los estudios realizados con una sola especie rara, tenemos diferentes enfoques que varían desde el estudio de las restricciones del hábitat (Prober y Austin, 1990), hasta el estudio de la biología de la reproducción (Wong y Sun, 1999) y de algunos parámetros demográficos (Schmalzel et al., 1995; Fisher y Matthies, 1998), incluyendo propuestas de estrategias de conservación (García, 1997). En estos trabajos se ha reportado que algunas especies raras presentan un alto nivel de especificidad de hábitat (Prober y Austin, 1990), mientras que en otros casos se reporta que poseen características reproductivas que tienen un efecto negativo en la tasa de crecimiento de la población, como un alto porcentaje de aborto y depredación de semillas, bajos niveles de fertilización, bajos porcentajes de germinación o serios problemas en el establecimiento (Godínez-Álvarez et al., 1999; EsparzaOlguín et al., 2002).

Los estudios uni-específicos generan información que, al ser específica y detallada, coadyuva a comprender algunos de los procesos involucrados en el fenómeno de la rareza en especies vegetales. Sin embargo, el conocimiento acerca de la dinámica poblacional de las especies raras sigue siendo muy pobre (Kunin y Gaston, 1997), debido particularmente a la falta de trabajos en los cuales se estudien las características demográficas y de dinámica poblacional de las especies raras, tales como las tasas de natalidad y mortalidad, la fecundidad, los estadios críticos a lo largo del ciclo de vida de las especies y la tasas de crecimiento poblacional. En opinión de muchos investigadores, son estas características las que determinan que ciertas especies presenten distribuciones geográficas restringidas o sean localmente poco abundantes (Lande, 1988; Menges, 1990; Schemske et al., 1994). De aquí la importancia de realizar análisis demográficos preferentemente comparativos en especies vegetales raras, que nos permitan conocer cómo se 


\section{Ligia Guadalupe EsParZa-Olguín}

comportan en las diferentes especies las tasas de natalidad y mortalidad, cómo es la reproducción, qué tan vulnerables son las diferentes etapas del ciclo de vida, y cómo varían estos atributos en el tiempo y en el espacio. Este tipo de estudios sobre la dinámica poblacional y la historia de vida de las especies nos permitirán conocer de qué manera su abundancia se ve regulada a partir de los elementos antes citados.

Los análisis demográficos en el estudio de la rareza. La demografía es una herramienta que nos permite conocer cómo y por qué cambia el tamaño de una población a través del tiempo. El estudio de la dinámica de una población aborda tres aspectos fundamentales que están, a su vez, íntimamente relacionados con la abundancia de las especies: 1) la tasa de crecimiento poblacional, determinada a partir de las tasas de natalidad, mortalidad, migración y emigración; 2) los atributos de la historia de vida de los organismos (e.g. edad a la primera reproducción, número de eventos reproductivos durante el ciclo de vida, número de descendientes en cada evento reproductivo, longevidad máxima, etc.); y 3) las proyecciones sobre el futuro de la población suponiendo que las condiciones iniciales permanecieran constantes (Franco, 1990; Stearns, 1992). Un estudio demográfico enfocado al análisis de los patrones generales de rareza debería considerar al menos los tres aspectos antes señalados (Kunin y Gaston, 1997; Bevill y Louda, 1999).

Se han desarrollado diferentes metodologías para estudiar la dinámica poblacional. Una de las más utilizadas en la actualidad son los modelos matriciales de proyección poblacional (Caswell 1989; Menges, 1990; Schemske et al., 1994). Los análisis matriciales representan una herramienta muy poderosa para el análisis del comportamiento demográfico de las especies raras, ya que permiten realizar proyecciones y simulaciones sobre el futuro potencial de sus poblaciones e identificar los estadios del ciclo de vida más vulnerables. Esta información, a su vez, puede integrarse a planes de manejo y conservación. Sin embargo, este tipo de análisis tienen ciertas restricciones que deben entenderse claramente para sacar el mayor provecho posible de los resultados obtenidos con estos modelos. Por ejemplo, los modelos matriciales convencionales son determinísticos y presuponen que las condiciones establecidas por la matriz se mantienen constantes, por lo que no consideran que el crecimiento poblacional puede estar afectado por cambios en el ambiente y por factores densodependientes, que llevan a variaciones en la mortalidad, la natalidad, el crecimiento y la reproducción de los individuos (Bierzychudek, 1982; Menges, 1990; Boyce, 1992).

Entre los ejemplos de estudios demográficos utilizando matrices de proyección y comparando las características de especies vegetales raras y comunes cercanamente emparentados se encuentra el de Fiedler (1987) y el de
Byers y Meagher (1997). Fiedler (1987) comparó tres especies raras del género Calochortus (C. obispoensis, $C$. tiburonesis y $C$. pulchellus) con una común, C. albus, durante un periodo de cuatro años (1981-84) en la costa central de California. Esta autora encuentra que, aunque en dos periodos de estudio (1982-83 y 1983-84) las tasas finitas de crecimiento poblacional $(\lambda)$ fueron mayores en $C$. albus (1.714 y 1.336) que en las especies raras (C. pulchellus 0.997/1.073, C. obispoensis 1.031/0.960 у $C$. tiburonensis 1.302/0.992), las poblaciones estudiadas de las cuatro especies estaban creciendo. A partir de análisis de sensibilidad, la autora sugiere que las especies raras tienen un comportamiento reproductivo "caótico", con ciertos periodos de alta producción de semillas que parecen asegurar su persistencia a largo plazo. Además, afirma que cada una de las especies raras estudiadas exhibe un comportamiento demográfico característico, que puede distinguirlas no sólo de la especie común, sino también de las otras especies raras. Finalmente, comenta que la rareza es una respuesta a muchos factores como las condiciones ambientales, las características biológicas inherentes a cada especie y los factores históricos, entre otros.

Por su parte, Byers y Meagher (1997) trabajaron con dos especies del género Eupatorium, E. perfoliatum (común) y E. resinosum (rara). Estos autores estudiaron tres poblaciones de cada una de las especies por tres años (1989-91) y encontraron que E. resinosum (rara) tiene niveles menores de reproducción sexual que E. perfoliatum (común), lo que aparentemente se refleja en un decrecimiento poblacional en el tiempo. Estos autores hacen énfasis en el hecho de que las especies raras y comunes cercanamente emparentadas pueden diferir drásticamente en sus estrategias de reproducción (i.e. reproducción sexual vs. propagación vegetativa) y señalan la importancia de conocer estos comportamientos para tomar las decisiones correctas en el terreno de la conservación.

Byers y Meagher (1997) mencionan que los valores de $\lambda$ que encontraron en su estudio (E. resinosum, la rara: 0.747 , 1.169 y 1.047 ; E. perfoliatum, la común: $1.088,0.764$ y 0.791 ) deben tomarse con precaución, puesto que en el caso de $E$. resinosum, $\lambda$ podría fluctuar drásticamente debido a cambios en las condiciones ambientales, ya que esta especie es propia de los primeras etapas serales de las comunidades en las que se establece y su persistencia está ligada a la ocurrencia de disturbios. Los autores concluyen que es necesario comparar los parámetros demográficos entre especies raras y comunes cercanamente emparentadas para entender el papel que juega la dinámica poblacional como causa de la rareza, señalando la importancia de tener réplicas de varias poblaciones de una misma especie. Por último, sugieren que esta información es indispensable para tomar decisiones correctas para la conservación o el manejo de las especies raras.

Los resultados de los dos trabajos aquí discutidos per- 
miten evidenciar ciertas coincidencias en el comportamiento demográfico de las especies analizadas, que son herbáceas perennes. En ambos estudios los valores de $\lambda$ se encuentran cercanos a la unidad y no son muy diferentes de los valores calculados para las especies comunes, y en ambos casos se enfatiza la importancia que tienen los cambios temporales en el ambiente sobre la fluctuación en el valor de $\lambda$. Los estudios coinciden también en la observación de que las especies raras tienden a mostrar una reproducción sexual más limitada que sus congéneres comunes. En un caso (Fiedler, 1987) se sugiere la posibilidad de un comportamiento "caótico" en los eventos de reproducción de las especies raras y en el otro (Byer y Meagher, 1997) se enfatiza la relevancia de la propagación asexual.

Entre los estudios demográficos con un enfoque uniespecífico, Schemske et al. (1994) incluyen en su revisión varios ejemplos. Además, tenemos ejemplos más recientes, como el trabajo realizado por Schmazel y colaboradores (1995) con Coryphantha robbinsorum (Cactaceae), en el que estudiaron tres poblaciones al sur de Arizona durante cinco años. Estos autores encontraron que existen asociaciones significativas entre diferentes eventos demográficos y las condiciones ambientales. Por ejemplo, los bajos porcentajes de reclutamiento de nuevos individuos estuvieron relacionados con periodos en los cuales la precipitación durante la temporada invernal de lluvia fue baja. Los autores reportan tasas finitas de crecimiento poblacional ligeramente mayores a uno para las tres poblaciones $(1.094,1.003$ y 1.041$)$ y señalan que las poblaciones presentan densidades bajas, por lo que sugieren que el valor de $\lambda$ podría decrecer con el tiempo; de ahí que, en su opinión, resulte indispensable continuar monitoreando las poblaciones estudiadas. Las simulaciones realizadas modificando la permanencia y la fecundidad muestran que el efecto más drástico se da al disminuir en un $50 \%$ la permanencia de los individuos adultos $(\lambda=0.822)$ y se concluye que una práctica recomendable para la conservación de esta especie consistiría en la protección de los individuos de esta categoría.

En el trabajo realizado por Esparza-Olguín et al. (2002), se estudió una población de Neobuxbaumia macrocephala, cactácea columnar endémica del Valle de Tehuacán, en el centro de México, durante dos periodos anuales (1997-98 y 1998-99). El porcentaje de germinación en condiciones naturales fue extremadamente bajo $(0.25 \%)$ y la probabilidad de establecimiento de las plántulas fluctuó entre 0.025 a 0.074. La tasa finita de crecimiento mostró valores por debajo de la unidad (aunque no de manera significativa: $0.979 \pm 0.119$ y $0.994 \pm 0.115)$. Al hacer simulaciones modificando las entradas de la matriz que corresponden a la permanencia, la fecundidad y el establecimiento, se encontró que el valor de $\lambda$ se reduce considerablemente al disminuir la permanencia de los individuos adultos en un
$30 \%(\lambda=0.931)$, mientras que al aumentar la fecundidad o el establecimiento hasta 10 veces se obtienen valores de $\lambda$ por encima de la unidad (1.002 en ambos casos). Se sugiere que la clave para asegurar la permanencia de esta especie está en la protección de los individuos adultos y en fomentar el establecimiento de las plántulas, de tal manera que aumente el reclutamiento en la población.

En el caso del trabajo reportado por Godínez-Álvarez et al. (1999), se hace un análisis de la dinámica poblacional de Neobuxbaumia tetetzo, cactácea columnar endémica de los estado de Puebla y Oaxaca, en México. En este caso los autores reportan que las fases más críticas del ciclo de vida de esta especie son la germinación y el establecimiento. Señalan que la polinización y la dispersión de semillas juegan un papel importante en la dinámica poblacional de $N$. tetetzo, la cual, además, presenta una reproducción intermitente, con años en los cuales prácticamente no se producen estructuras reproductoras. La tasa de crecimiento poblacional que encuentran estos autores es igual a la unidad.

Aunque los ejemplos referidos en los párrafos anteriores no cubren de manera exaustiva la literatura sobre demografía de especies raras, sí nos permiten ver algunos rasgos en común entre las especies estudiadas, las cuales coinciden en presentar bajas tasas de reclutamiento, asociadas a condiciones ambientales estresantes que parecen jugar un papel preponderante (i.e. radiación intensa, escasa precipitación, etc.). Otra coincidencia importante radica en los valores de $\lambda$ obtenidos a partir del análisis de las matrices: en los tres casos los valores de $\lambda$ son muy cercanos a la unidad. Los resultados de las simulaciones realizadas por Schmazel et al. (1995) y por Esparza-Olguín et al. (2002) muestran, por un lado, lo difícil que es lograr aumentos en el valor de la tasa finita de crecimiento poblacional y, por otro lado, la gran vulnerabilidad de estas poblaciones, pues el valor de $\lambda$ decrece fácilmente. Estos resultados indican que algunas especies raras presentan limitaciones inherentes a su biología que impiden un aumento significativo en el crecimiento poblacional. También hacen notar que las condiciones ambientales (i.e. cuando se presentan periodos favorables sólo ocasionalmente) tienen una fuerte influencia en la dinámica poblacional de estas especies. Sin embargo, nuevamente el bajísimo número de estudios hasta ahora publicados para especies raras donde se señalen por lo menos los valores de las tasas de crecimiento poblacional y algunos de los estadios más vulnerables del ciclo de vida de las especies, hace prácticamente imposible saber en qué medida el comportamiento de las especies raras citado en estos ejemplos constituye un patrón general.

La genética de poblaciones en el estudio de la rareza. En el campo de la genética de poblaciones se han llevado a cabo un gran número de estudios en los cuales se aborda el estudio de la variabilidad genética en poblaciones de especies raras. Esto se debe a que, como se dijo en un prin- 


\section{Ligia GuAdALUPE EsPaRZA-Olguín}

cipio, el estudio de la rareza como fenómeno biológico ha estado ligado a la biología de la conservación y en este sentido se reconoce que la conservación a largo plazo de las especies raras depende de nuestra capacidad de conocer y entender los procesos evolutivos a los que están sometidas (Hamrick y Godt, 1996).

$\mathrm{El}$ estudio de la diversidad genética en poblaciones naturales de especies vegetales raras se ha abordado utilizando diferentes técnicas moleculares. Durante la década de 1980 y principios de la década de 1990 la técnica molecular más utilizada fue la electroforesis de isoenzimas. Los estudios realizados con esta técnica permitieron conocer los patrones de variabilidad genética inter- e intra-poblacional y así examinar el impacto de los procesos de dispersión y de los sistemas reproductivos en la diversidad genética de un gran número de especies (Cruzan, 1998). Los resultados de estos estudios han revelado, en su mayoría, que las especies que se distribuyen a manera de poblaciones pequeñas o aisladas (es decir, que son raras) presentan una baja diversidad genética (cuadro 1).
Una de las especies estudiadas bajo este enfoque fue Gentiana pneumonanthe, hierba perenne de vida larga, autoincompatible con polinización cruzada. Para esta especie se reportó una heterocigosidad observada de Ho $=$ 0.18 , lo cual se considera un índice bajo, en comparación con otras especies más comunes (Oostermeijer et al., 1994). El análisis con isoenzimas reveló que la pérdida de grandes extensiones de hábitat (a causa de la extensión de la frontera agrícola) ha reducido drásticamente las poblaciones de esta especie, aumentando los niveles de endogamia y reduciendo el número de heterócigos en sus poblaciones. Aparentemente esto ha tenido el efecto de reducir la adecuación a nivel poblacional. Otro estudio de este tipo es el realizado con Lynchnis viscaria (Lammí et al., 1999), para la cual se evalúo la variación genética en poblaciones periféricas y poblaciones centrales con respecto a su área de distribución en Finlandia. En ambos tipos de poblaciones se encontraron niveles bajos de variabilidad genética (periféricas $\mathrm{H}=0.034$ y centrales $\mathrm{H}=0.114$ ) pero, contrario a lo esperado, no hubo diferencias significativas

Cuadro 1. Información general sobre la variabilidad genética en especies vegetales raras. Abreviaturas: \% $\mathrm{P}=$ Porcentaje de polimorfismo, $\mathrm{A}=$ Número promedio de alelos por locus, $\mathrm{Ho}=$ Heterocigosis promedio observada, $\mathrm{HE}_{\mathrm{E}}=$ Heterocigosis promedio esperada, $\mathrm{H}_{\mathrm{T}}=\mathrm{Diversidad}$ genética total, $\mathrm{Hs}_{\mathrm{s}}=$ Variación genética promedio dentro de las poblaciones, Fst/Gst = Coeficientes de diferenciación genética entre poblaciones, $\mathrm{V}=$ Variabilidad exhibida en la población.

* Datos provenientes de análisis con isoenzimas. + Datos provenientes de análisis con RAPDs.

\begin{tabular}{|c|c|c|c|c|c|c|c|c|c|}
\hline Familia y especie & $\% \mathbf{P}$ & A & Ho & $\mathrm{HE}_{\mathrm{E}}$ & $\mathbf{H}_{\mathrm{T}}$ & $\mathrm{Hs}_{\mathrm{s}}$ & Fst/Gst & $\mathbf{V}$ & Referencias \\
\hline \multicolumn{10}{|l|}{ Agavaceae } \\
\hline Agave cerulata & 77.6 & & & 0.181 & & 0.181 & 0.098 & & Navarro-Quezada (1999) \\
\hline Agave subsimplex & 78.5 & & & 0.142 & & 0.143 & 0.084 & & Navarro-Quezada (1999) \\
\hline Agave victoriae-reginae & 83 & 2.2 & & 0.335 & & & 0.236 & & Martínez-Palacios et al. (1999) \\
\hline \multicolumn{10}{|l|}{ Alismataceae } \\
\hline Sagitaria isoetiformis & 93.8 & 1.90 & & 0.218 & & & 0.399 & & Edward y Sharitz (2000) \\
\hline Sagitaria teres & 75 & 1.30 & & 0.101 & & & 0.261 & & Edward y Sharitz (2000) \\
\hline \multicolumn{10}{|l|}{ Alliaceae } \\
\hline Allium aaseae & 56.9 & & & & 0.323 & 0.274 & 0.126 & & Smith y Pham (1996) \\
\hline \multicolumn{10}{|l|}{ Anacardiaceae } \\
\hline Rhus michauxii & 47 & & 0.07 & & 0.215 & & 0.335 & & Sherman-Broyles et al. (1992) \\
\hline \multicolumn{10}{|l|}{ Apiaceae } \\
\hline Aletes humilis & 81 & 303 & & & 0.327 & 0.249 & 0.197 & & Linhart y Premoli (1993) \\
\hline Lomatium rollinsii & 18.4 & 1.22 & & & & & & & Soltis et al. (1997) \\
\hline Lomatium serpentinum & 2.8 & 1.03 & & & & & & & Soltis et al. (1997) \\
\hline Lomatium laevigatum & 2.6 & 1.02 & & & & & & & Soltis et al. (1997) \\
\hline \multicolumn{10}{|l|}{ Asteraceae } \\
\hline $\begin{array}{l}\text { Achillea millefolium } \\
\text { var. megacephala }\end{array}$ & 31.6 & 1.45 & 0.129 & 0.126 & 0.296 & 0.272 & 0.078 & & Purdy y Bayer (1996) \\
\hline Antennaria virginica & 22.9 & 1.27 & 0.093 & & 0.107 & & 0.187 & & Bayer y Crawford (1986) \\
\hline $\begin{array}{l}\text { Argyroxiphium sandwicense } \\
\text { var. sandwicense }\end{array}$ & 2.5 & & & & & 0.183 & & & Friar et al. (1996) \\
\hline Centaurea corymbosa & 11.8 & 1.1 & 0.043 & & & & 0.334 & & Fréville et al. (1998) \\
\hline Centaurea maculosa var. albida & 23.5 & 1.5 & 0.071 & & & & & & Fréville et al. (1998) \\
\hline
\end{tabular}


RAREZA EN ESPECIES VEGETALES: ENFOQUE GENÉTICO-DEMOGRÁFICO

Cuadro 1. Continuación

\begin{tabular}{|c|c|c|c|c|c|c|c|c|c|}
\hline Familia y especie & $\% P$ & A & Ho & $\mathrm{HE}_{\mathrm{E}}$ & $\mathbf{H}_{\mathbf{T}}$ & $\mathrm{Hs}_{\mathrm{s}}$ & Fst/Gst & $\mathbf{V}$ & Referencias \\
\hline Cirsium pitcheri & 28.6 & 2.5 & 0.018 & 0.024 & 0.119 & 0.083 & 0.139 & & Loveless y Hamrick (1988) \\
\hline Dendroseris verteroana & & & & & 0.000 & & & & Esselman et al. (2000) \\
\hline Dendroseris litoralis & & & & & 0.071 & & & & Esselman et al. (2000) \\
\hline Dendroseris micrantha & & & & & 0.065 & & & & Esselman et al. (2000) \\
\hline Dendroseris neriifolia & & & & & 0.053 & & & & Esselman et al. (2000) \\
\hline Dendroseris pinnata & & & & & 0.024 & & & & Esselman et al. (2000) \\
\hline Dendroseris pruinara & & & & & 0.000 & & & & Esselman et al. (2000) \\
\hline Echinacea tennesseensis & 27.8 & 1.5 & 0.069 & & & & 0.02 & & Baskauf y Eickmeier (1994) \\
\hline Hymenopappus artemisifolius & 71 & 2.05 & 0.208 & & 0.371 & 0.311 & 0.162 & & Babbel y Selander (1974) \\
\hline Layia discoidea & 90.5 & 3.1 & & & & & & & Gottlieb et al. (1985) \\
\hline Layia jonesii & 64.7 & 3.0 & & & & & & & Warwick y Gottlieb (1985) \\
\hline Layia munzii & 52.9 & 3.2 & & & & & & & Warwick y Gottlieb (1985) \\
\hline Layia leucoppa & 58.8 & 3.2 & & & & & & & Warwick y Gottlieb (1985) \\
\hline Lasthenia maritima & 8.9 & 2 & & & 0.104 & 0.024 & 0.294 & & Crawford et al. (1985) \\
\hline $\begin{array}{l}\text { Stephanomeria exigua } \\
\text { var. carotufera }\end{array}$ & 57 & 2.10 & 0.092 & & 0.331 & 0.300 & 0.077 & & Hamrick (1983) \\
\hline $\begin{array}{l}\text { Stephanomeria exigua } \\
\text { var. coronaria }\end{array}$ & 71.4 & 2.3 & & & & & & & Gottlieb (1973a,b, 1977) \\
\hline Tragopogon minus & 4.2 & & 0.430 & & 0.188 & 0.038 & 0.515 & & $\begin{array}{l}\text { Roose y Gottlieb (1976) } \\
\text { Hamrick (1983) }\end{array}$ \\
\hline Tragopogon miscellus & & & & & 0.154 & 0.133 & 0.136 & & $\begin{array}{l}\text { Roose y Gottlieb (1976) } \\
\text { Hamrick (1983) }\end{array}$ \\
\hline Wyethia reticulata & $\begin{array}{l}67^{*} \\
68.7\end{array}$ & $1.87^{*}$ & 0.240 & 0.240 & 0.280 & & $\begin{array}{l}0.250^{*} \\
0.273+\end{array}$ & & Ayres y Ryan (1997) \\
\hline \multicolumn{10}{|l|}{ Brassicaceae } \\
\hline Warea carteri & & 1.87 & 0.018 & 0.026 & & & 0.304 & & Evans et al. (2000) \\
\hline \multicolumn{10}{|l|}{ Cactaceae } \\
\hline Carnegiea gigantea & 53.7 & 2.20 & 0.110 & 0.116 & & & 0.075 & & Hamrick et al. (2002) \\
\hline Lophocereus schottii & 49.5 & 2.33 & 0.142 & 0.144 & & & 0.242 & & Hamrick et al. (2002) \\
\hline Pachycereus pringlei & 62.1 & 2.50 & & 0.200 & & & 0.076 & & Hamrick et al. (2002) \\
\hline Pereskia guamacho & 63.4 & 2.42 & 0.169 & 0.202 & & & 0.112 & & Hamrick et al. (2002) \\
\hline Stenocereus eruca & 46.2 & 1.48 & 0.040 & 0.154 & & & 0.069 & & Clark-Tapia (2000) \\
\hline Stenocereus griceus & 57.1 & 2.36 & 0.139 & 0.167 & & & 0.096 & & Hamrick et al. (2002) \\
\hline Stenocereus gummosus & 75 & 1.96 & 0.103 & 0.261 & & & 0.102 & & Clark-Tapia (2000) \\
\hline Stenocereus thurberi & 62.4 & 2.36 & 0.157 & 0.169 & & & 0.128 & & Hamrick et al. (2002) \\
\hline \multicolumn{10}{|l|}{ Caryophyllaceae } \\
\hline $\begin{array}{l}\text { Cerastium fischerianum } \\
\text { var. molle }\end{array}$ & & & & & 0.479 & 0.273 & & & Maki y Horie (1999) \\
\hline Lychnis viscaria & 21 & 1.3 & 0.050 & 0.056 & & & 0.430 & & Lamni et al. (1999) \\
\hline Stellaria arenicola & 30 & 1.41 & & 0.087 & 0.163 & 0.147 & 0.102 & & Pudrí y Macdonald (1994) \\
\hline \multicolumn{10}{|l|}{ Ceratophylaceae } \\
\hline Ceratophylium echinatum & 7 & 1.07 & 0.071 & & 0.234 & & 0.845 & & Les (1991) \\
\hline \multicolumn{10}{|l|}{ Convallariaceae } \\
\hline Tricyrtis flava & 64.7 & 2.05 & & & 0.168 & & 0.409 & & Maki y Horie (1999) \\
\hline \multicolumn{10}{|l|}{ Dipsacadeae } \\
\hline Scabiosa columbaria & & & & & & & & & Bijisma et al. (1991) \\
\hline \multicolumn{10}{|l|}{ Fabaceae } \\
\hline Astragalus linifolius & 64 & 1.42 & 0.375 & 0.440 & & & 0.020 & & Karron et al. (1988) \\
\hline Astragalus osterhouti & 79 & 1.25 & 0.325 & 0.357 & & & & & Karron et al. (1988) \\
\hline Baptisia nuttaliana & 20 & 1.20 & 0.275 & & & & & & Hamrick et al. (1979) \\
\hline Lespedeza leptostachya & 0 & 1.0 & 0.000 & & & & 0.000 & & Cole y Biesboer (1992) \\
\hline Lupinus nanus & 50 & 1.50 & 0.241 & & & & & & Scogin $(1973)$ \\
\hline Lupinus subcarnosus & 88 & 1.84 & 0.241 & & 0.913 & 0.119 & 0.383 & & $\begin{array}{l}\text { Babbel y Selander (1974) } \\
\text { Hamrick (1983) }\end{array}$ \\
\hline Lupinus texensis & 87.5 & 3.12 & 0.356 & & 0.525 & 0.428 & 0.183 & & $\begin{array}{l}\text { Babbel y Selander (1974) } \\
\text { Hamrick (1983) }\end{array}$ \\
\hline
\end{tabular}


Ligia GuAdALUPE ESPARZA-Olguín

Cuadro 1. Continuación

\begin{tabular}{|c|c|c|c|c|c|c|c|c|c|}
\hline Familia y especie & $\% \mathbf{P}$ & A & Ho & $\mathrm{HE}_{\mathrm{E}}$ & $\mathbf{H}_{\mathbf{T}}$ & $\mathrm{Hs}$ & Fst/Gst & $\mathbf{V}$ & Referencias \\
\hline \multicolumn{10}{|l|}{ Gentianaceae } \\
\hline Gentiana pneumonanthe & & & 0.184 & & & & & & Oostermeijer et al. (1994) \\
\hline Lisianthus aurantiacus & 33.3 & 2 & 0.106 & & & & & & Systma y Schaal (1985) \\
\hline Lisianthus habuansis & 8.3 & 2 & 0.017 & & & & & & Systma y Schaal (1985) \\
\hline Lisianthus jefensis & 16.7 & 2 & 0.03 & & & & & & Systma y Schaal (1985) \\
\hline Lisianthus peduncularis & 33.3 & 2 & 0.073 & & & & & & Systma y Schaal (1985) \\
\hline \multicolumn{10}{|l|}{ Grammitidaceae } \\
\hline Adenophorus periens & 80 & 2.8 & 0.191 & & & & & & Ranker (1994) \\
\hline \multicolumn{10}{|l|}{ Labiatae } \\
\hline Agastache cusickii & 60 & 1.80 & 0.166 & & & & & & Vogelman y Gastony (1987) \\
\hline Agastache occidentalis & 66.7 & 2.07 & 0.122 & & & & & & Vogelman y Gastony (1987) \\
\hline Agastache parvifolia & 53.3 & 1.80 & 0.107 & & & & & & Vogelman y Gastony (1987) \\
\hline \multirow[t]{2}{*}{ Salvia pratensis } & 31 & 1.34 & & & & & 0.156 & & Bijisma et al. (1991) \\
\hline & & & & & & & & & Ouborg y van Treuren (1995) \\
\hline \multicolumn{10}{|l|}{ Lauraceae } \\
\hline Lindera melissifolia & 33.3 & 1.46 & & & 0.020 & & & & Godt y Hamrick (1996) \\
\hline \multicolumn{10}{|l|}{ Liliaceae } \\
\hline Erythronium propullans & 24 & 1.80 & 0.180 & 0.33 & & & & & Pleasants y Wedel (1989) \\
\hline Helonias bullata & 12.8 & 1.14 & 0.025 & 0.029 & 0.160 & 0.086 & 0.306 & & Godt et al. (1995) \\
\hline Helonias hakuuenensis & 76.2 & 2.99 & 0.188 & 0.245 & & & 0.249 & & Godt et al. (1995) \\
\hline Helonias hongdoensis & 55.6 & 2.11 & 0.119 & 0.165 & & & 0.269 & & Godt et al. (1995) \\
\hline Helonias taeanensis & 81.3 & 3.21 & 0.151 & 0.231 & & & 0.411 & & Godt et al. (1995) \\
\hline \multicolumn{10}{|l|}{ Limnanthaceae } \\
\hline Limnanthes alba & 52.2 & 1.73 & 0.159 & & & & & & Arroyo (1975) \\
\hline Limnanthes bakeri & 36.8 & 2.3 & & & & & & & Kesseli y Jain (1984) \\
\hline Limnanthes floccosa & 17.6 & 1.19 & 0.130 & & & & & & Arroyo (1975) \\
\hline Limnanthes macounii & 11.1 & 2.0 & & & & & & & Kesseli y Jain (1984) \\
\hline Limnanthes vinculans & 41.2 & 3.0 & & & & & & & Keseli y Jain (1984) \\
\hline \multicolumn{10}{|l|}{ Malvaceae } \\
\hline Hibiscus dasycalyx & 10 & & & & & & & & Clips (1995) \\
\hline \multicolumn{10}{|l|}{ Myrtaceae } \\
\hline Austromytus gonoclada & 59.4 & 1.8 & 0.055 & & & & & & Shapcott y Playford (1996) \\
\hline Austromytus hillii & 31.3 & 1.4 & 0.010 & & & & & & Shapcott y Playford (1996) \\
\hline Austromytus inophloia & 31.3 & 1.6 & 0.025 & & & & & & Shapcott y Playford (1996) \\
\hline Eucalyptus argutifolia & 45.9 & 1.8 & 0.190 & & 0.261 & & 0.212 & & Kennington y James (1998) \\
\hline Eucalyptus caesia & 38.8 & 2.1 & & & & & & & Moran y Hoper (1983) \\
\hline Eucalyptus phylacis & & & & & & & & 0.00 & Rosseto et al. (1999) \\
\hline \multicolumn{10}{|l|}{ Onagraceae } \\
\hline Clarkia amoena & 62 & 1.37 & & & & & & & Gottlieb $(1973 a, b)$ \\
\hline Clarkia biloba & 61 & 2.09 & 0.150 & & & & & & Gottlieb (1974) \\
\hline Clarkia dudleyana & 75 & 2.09 & 0.160 & & & & & & Gottlieb (1974) \\
\hline Clarkia franciscana & 7.7 & 2.0 & 0.000 & & & & & & Gottlieb $(1973 a, b)$ \\
\hline Clarkia lingulata & 56 & 2.06 & 0.080 & & & & & & Gottlieb (1974) \\
\hline Clarkia rubicunda & 59 & 1.69 & 0.110 & & & & & & Gottlieb $(1973 a, b)$ \\
\hline Gaura demareei & 22 & 1.39 & 0.051 & & 0.198 & 0.186 & 0.060 & & Gottlieb y Plitz (1976) \\
\hline Oenothera argillicola & 20 & 1.25 & 0.080 & & & & & & Levy y Levin (1975) \\
\hline Oenothera organensis & 6.7 & 2.0 & & & & & & & Levy et al. (1979) \\
\hline \multicolumn{10}{|l|}{ Orchidaceae } \\
\hline \multirow[t]{2}{*}{ Goodyera procera } & $22^{*}$ & $1.22 *$ & $0.073^{*}$ & & & & $0.520^{*}$ & & Wong y Sun (1999) \\
\hline & $55+$ & & & & $0.290+$ & $0.180+$ & $0.390+$ & & \\
\hline \multicolumn{10}{|l|}{ Papaveraceae } \\
\hline Arctomecon humilis & 10.4 & 1.43 & 0.100 & & & & & & Allphin et al. (1998) \\
\hline
\end{tabular}


RAREZA EN ESPECIES VEGETALES: ENFOQUE GENÉTICO-DEMOGRÁFICO

Cuadro 1. Continuación

\begin{tabular}{|c|c|c|c|c|c|c|c|c|c|}
\hline Familia y especie & $\% \mathbf{P}$ & A & Ho & $\mathrm{He}_{\mathrm{E}}$ & $\mathbf{H}_{\mathbf{T}}$ & $\mathrm{Hs}_{\mathrm{s}}$ & Fst/Gst & v & Referencias \\
\hline \multicolumn{10}{|l|}{ Pinaceae } \\
\hline Picea engelmannii & & & & & & & 0.100 & & Hiebert y Hamrick (1983) \\
\hline Pinus balbouriana & 56.7 & 2.10 & & & & & & & Hiebert y Hamrick (1983) \\
\hline Pinus edulis & & 1.8 & & 0.150 & & & 0.012 & & Betancourt et al. (1991) \\
\hline Pinus longaeva & 78.6 & 2.35 & 0.327 & & 0.484 & 0.465 & 0.038 & & Hamrick (1983) \\
\hline Pinus pungens & 4 & 1.33 & 0.002 & & 0.342 & 0.331 & 0.032 & & Feret (1974) \\
\hline Pinus radiata & 45.4 & 2.2 & & & 0.154 & 0.133 & 0.136 & & $\begin{array}{l}\text { Moran et al. (1980) } \\
\text { Hamrick (1983) }\end{array}$ \\
\hline Pinus rzedowskii & 46.8 & 1.8 & 0.162 & 0.219 & & & 0.175 & & Delgado et al. (1999) \\
\hline Pinus torreyana & 3.4 & 2.2 & & & & & 1.000 & & Leding y Conkle (1983) \\
\hline Pinus washoensis & & & & 0.150 & & & 0.016 & & Niebling y Conkle (1990) \\
\hline \multicolumn{10}{|l|}{ Poaceae } \\
\hline Deschampsia mackenzieana & 18.9 & 1.55 & & & 0.189 & & 0.268 & & Purdy y Bayer (1995b) \\
\hline Elymus hystrix & 7.7 & & & & & & & & Clegg et al. (1976) \\
\hline Elymus wiegandii & 7.7 & & & & & & & & Clegg et al. (1976) \\
\hline \multicolumn{10}{|l|}{ Polemoniaceae } \\
\hline Phlox cuspidata & 7.9 & 1.08 & 0.010 & & 0.074 & 0.046 & 0.299 & & $\begin{array}{l}\text { Levin }(1975,1978) \\
\text { Hamrick }(1983)\end{array}$ \\
\hline Phlox roemariana & 16.3 & 1.13 & 0.046 & & 0.356 & 0.278 & 0.244 & & $\begin{array}{l}\text { Levin }(1975,1978) \\
\text { Hamrick }(1983)\end{array}$ \\
\hline \multicolumn{10}{|l|}{ Polygonaceae } \\
\hline Polygonella basiramia & 28.5 & 2.5 & & & & & & & Lewis y Crawford (1995) \\
\hline Polygonella ciliata & 21.3 & 2.0 & & & & & & & Lewis y Crawford (1995) \\
\hline Polygonella fimbriata & 33.3 & 2.28 & & & & & & & Lewis y Crawford (1995) \\
\hline Polygonella macrophylla & 29.6 & 2.27 & & & & & & & Lewis y Crawford (1995) \\
\hline Polygonella parksii & 23.7 & 2.55 & & & & & & & Lewis y Crawford (1995) \\
\hline Polygonella robusta & 54.5 & 2.17 & & & & & & & Lewis y Crawford (1995) \\
\hline \multicolumn{10}{|l|}{ Proteaceae } \\
\hline Grevillea scapigera & 4.3 & & & & & & & & Rossetto et al. (1995) \\
\hline Lambertia orbifolia & 38.5 & 1.5 & 0.060 & 0.100 & & & 0.441 & & Coates y Hamley (1999) \\
\hline Leucadendron elimense & & & & & & & & 0.35 & Tansley y Brown (2000) \\
\hline \multicolumn{10}{|l|}{ Rosaceae } \\
\hline Geum radiatum & 23 & & 0.052 & 0.074 & & & & & Hamrick y Godt (1996) \\
\hline \multicolumn{10}{|l|}{ Rutaceae } \\
\hline Zieria prostrata & 37 & & & & & & & & Hogbin y Peakall (1999) \\
\hline \multicolumn{10}{|l|}{ Salicaceae } \\
\hline Salíx salicifola & 35.3 & 1.59 & & & 0.305 & & 0.159 & & Purdy y Bayer (1995a) \\
\hline \multicolumn{10}{|l|}{ Sarraceniaceae } \\
\hline Sarracenia rubra var. alabamensis & 53.3 & 1.73 & 0.183 & & 0.262 & & 0.086 & & Godt y Hamrick (1998) \\
\hline \multicolumn{10}{|l|}{ Scrophulariaceae } \\
\hline Mabrya acerifolia & 15.9 & 1.16 & 0.041 & & 0.077 & & 0.208 & & Elisens y Crawford (1988) \\
\hline Mabrya coccinea & 9 & 1.09 & 0.035 & & & & & & Elisens y Crawford (1988) \\
\hline Mabrya rosei & 18.2 & 1.18 & 0.045 & & & & & & Elisens y Crawford (1988) \\
\hline Pedicularis furbishiae & 0 & 0 & 0.000 & 0.000 & & & & & Waller et al. (1987) \\
\hline \multicolumn{10}{|l|}{ Solanaceae } \\
\hline Capsicum cardenasii & 19.2 & 1.27 & & & & & & & McLeod et al. (1983) \\
\hline Lycopersicon parviflorum & 0 & 1 & 0.000 & & & & & & Rick et al. (1976) \\
\hline Lycopersicon pimpinellifolium & 36.3 & 1.54 & 0.074 & & 0.239 & 0.125 & 0.317 & & $\begin{array}{l}\text { Rick et al. (1977) } \\
\text { Hamrick (1983) }\end{array}$ \\
\hline
\end{tabular}


entre los niveles de adecuación de ambos tipos de poblaciones, encontrándose además niveles altos de diferenciación interpoblacional. Esto llevó a los autores a sugerir que la mejor estrategia de conservación para esta especie sería conservar no sólo las poblaciones centrales, sino también las poblaciones pequeñas y más aisladas (periféricas) con el fin de conservar la mayor cantidad de variabilidad genética posible.

A mediados de la década de 1990 se empezó a generalizar el uso de marcadores moleculares de ADN, como los microsatélites, los AFLPs y los RAPDs. Estas nuevas técnicas necesitan menos material y son más finas; además, permiten detectar niveles de variabilidad genética que las isoenzimas no detectan (cuadro 1) (Rossetto et al., 1995, Maki y Horie, 1999). En el caso de los estudios realizados con especies vegetales raras, el marcador molecular más utilizado han sido los RAPDs (Random Amplified Polymorfic DNA). Por ejemplo, en el estudio realizado por Friar et al. (1996) con Argyroxiphium sandwicense var. sandwicense se empleó esta técnica. Esta especie, endémica del volcán Mauna Kea en Hawaii, es autoincompatible y pasó por un cuello de botella que redujo la población de aproximadamente 40,000 individuos a apenas 4,000. Esto trajo como consecuencia la pérdida de variabilidad genética, presentando una heterocigosidad de apenas 0.17 , con un nivel de polimorfismo de 0.024. En la actualidad la población se reduce a tan solo 46 individuos. Otro ejemplo es el estudio realizado por Hogbin y Peakall (1999), en el cual se analiza la variabilidad genética en poblaciones de Zieria prostrata. Esta especie arbustiva postrada presenta altos niveles de autofertilización en poblaciones semiaisladas. El análisis con RAPDs mostró niveles de variabilidad altos $(65 \%)$ a nivel intrapoblacional, pero niveles bajos de variabilidad $(37 \%)$ a nivel interpoblacional.

La mayoría de los estudios que han evaluado la variabilidad genética intra- o interpoblacional en poblaciones naturales de especies raras (cuadro 1) han encontrado poca o nula variabilidad en estas especies. Sin embargo, existen reportes de especies raras con alta variabilidad genética (cuadro 1) y de especies muy comunes con muy poca variabilidad genética (e.g. Pteridium aquilinum) (Gottilieb et al., 1985, Karron et al., 1988, Ranker, 1994, Lewis y Crawford, 1995, Smith y Pham, 1996). Por ejemplo, en Grevillea scapigera, especie endémica de una pequeña región de Australia y hasta hace poco considerada extinta, se detectó un nivel relativamente alto de variabilidad genética ( $\mathrm{V}=0.32$; Rossetto et al., 1995). El $87 \%$ de esta variabilidad corresponde a variabilidad intrapoblacional, mientras que el restante $13 \%$ se refiere a variabilidad interpoblacional. Los autores señalan que esto se debe probablemente a que ésta es una planta exógama. En Allium aaseae, especie endémica del suroeste de Idaho, también se encontraron altos niveles de variación genética $(\mathrm{Ht}=0.446$, $\mathrm{p}=0.569$; Smith y Pham, 1996); los autores señalan que estos resultados son en parte el reflejo de los altos niveles de entrecruzamiento entre individuos y entre poblaciones de esta especie. Sugieren además, como hipótesis alternativa, que es posible que esta especie tenga poco tiempo de haber divergido de su ancestro A. simillimum, que también tiene altos niveles de variación genética $(\mathrm{H}=0.500, \mathrm{p}=$ $0.590)$, por lo que tal vez aún conserve gran parte de dicha variación. Una alternativa es que exista hibridización entre ambas especies, a consecuencia de la cual se integren constantemente a la población de $A$. aaseae nuevos alelos.

Gitzendanner y Soltis (2000) publicaron una revisión de los patrones de variación genética en especies raras y comunes cercanamente emparentadas y señalaron que las revisiones de este tipo publicadas previamente (e.g. Hamrick et al., 1979; Stebbins, 1980; Hamrick y Godt, 1989) no consideran la importancia de las relaciones filogenéticas entre las especie estudiadas. Los autores señalan que este "olvido" viola algunos de los supuestos de los métodos estadísticos utilizados para analizar los datos, por lo que no es posible afirmar que los patrones reportados en esas publicaciones sean del todo válidos. En esta nueva revisión los autores se dan a la tarea de buscar patrones en el comportamiento de especies raras, a partir de datos publicados, por medio de la comparación de sus características a nivel genético con especies comunes congéneres cercanamente emparentadas. Estos autores encuentran que, efectivamente, las especies vegetales raras presentan en general menor variabilidad genética que sus congéneres comunes, sin que esto signifique necesariamente que tengan poca variabilidad genética $\left(\% \mathrm{P}_{\text {raras }}=36.7, \% \mathrm{P}_{\text {comunes }}=44.9\right.$, Horaras $=0.095$, Hocomunes $=0.119, \mathrm{H}_{\text {Traras }}=0.219, \mathrm{H}_{\text {Tcomunes }}=$ 0.242). Este resultado concuerda con el reportado por Young y Brown (1996), quienes concluyen que en algunos géneros tanto las especies raras como las comunes presentan muy altos o muy bajos niveles de polimorfismo, siendo éste muy parecidos en ambos tipos de especies.

La gama de valores de variabilidad genética encontrada en las especies vegetales raras es hasta el momento muy amplia. Existen especies raras con altos niveles de variabilidad genética, por ejemplo, Agastache occidentalis (Vogelmann y Gastony, 1987), Adenophorus periens (Ranker, 1994) y Daviesia suaveolens (Young y Brown, 1996), y otras con bajos niveles de variabilidad genética, por ejemplo, Ceratophyllum echinatum (Les, 1991), Bensoniella oregona (Soltis et al., 1992) y Lespedeza leptostachya (Cole y Biesboer, 1992).

\section{Discusión}

La información demográfica de la que se dispone hasta la fecha para diferentes especies vegetales raras nos permite observar ciertos patrones demográficos que, sin embargo, por el momento no podemos considerar como generales en este tipo de especies, debido principalmente a la escasez de 
estudios de este tipo. Hasta ahora todas las especies de plantas raras que se han estudiado a este nivel presentan tasas de crecimiento poblacional cercanas a la unidad. Además, en su mayoría tienen limitaciones para reproducirse sexualmente y, en algunos casos, la reproducción presenta un comportamiento intermitente, con periodos largos de baja reproducción sexual, seguidos por periodos cortos en los que la reproducción sexual es relativamente más exitosa. Sin embargo, no podemos decir que estos comportamientos constituyan patrones demográficos generales en las especies raras, pues algunas de estas características demográficas se presentan también en especies relativamente más comunes. Esto mismo sucede con los reportes de bajos porcentajes de germinación y bajas tasas de reclutamiento de nuevos individuos en las poblaciones de las especies raras. Todas estas características inciden directamente en la abundancia y en la distribución de las especies, por lo que pueden considerarse causas o consecuencias de la rareza, ya que en todos los casos limitan tanto la abundancia como la distribución de las especies. En este contexto, es importante reconocer que las especies raras no constituyen un ensamble uniforme, sino que, por el contrario, representan una amplia gama de taxones con ca-racterísticas biológicas diversas, en los que cada especie posee sus propios atributos demográficos y está representada por poblaciones locales que presentan abundancias diversas, distintos niveles de especificidad de hábitat y áreas de distribución geográfica heterogéneas, lo que se traduce en diferentes niveles e incluso tipos de rareza (Rabinowitz, 1981).

En lo que a los estudios genéticos se refiere, la gran cantidad de trabajos publicados sobre el tema muestra claramente la importancia de considerar las diferencias y afinidades ecológicas y filogenéticas al comparar especies raras, con el objeto de poder evaluar de manera adecuada la existencia potencial de patrones generales para este tipo de especies. Queda de manifiesto que la comparación del comportamiento demográfico y de las características genéticas entre especies congéneres raras y comunes es crucial para la identificación y el entendimiento de las causas evolutivas y ecológicas de la rareza y que, más que proponer generalizaciones sobre la biología de las especies raras, es indispensable enfocar los esfuerzos de investigación hacia tratar de entender y explicar cómo se relacionan diferentes características ecológicas y evolutivas, y de qué manera podemos concebirlas como causas o consecuencias de la rareza en especies vegetales. En este sentido, es notable la ausencia de estudios que incorporen simultáneamente aspectos genéticos y ecológicos, en un intento por comprender el fenómeno de la rareza desde ambas perspectivas. A pesar de que esta falta de articulación entre la genética de poblaciones y la demografía se ha mencionado en repetidas ocasiones (van Groenendael et al., 1988; Silvertown, 1991; Schemske et al., 1994), es claro que a la fecha todavía no se ha construido un puente tangible entre ambas disciplinas. El conocimiento que se podría generar de un enfoque interdisciplinario de esta naturaleza nos permitirá no sólo ampliar nuestro conocimiento y comprensión sobre la biología de las especies raras, sino que también nos ayudará a generara mejores propuestas de conservación para éstas.

En este sentido, valdría la pena considerar cómo es la cadena causal de la rareza en cada especie. ¿Es posible que cada especie tenga su propia cadena causal o, por el contrario, que podamos encontrar patrones generales en este sentido? Por ejemplo, considerando que tanto la distribución como la abundancia de las poblaciones están determinadas primordialmente por parámetros demográficos, como las tasas de natalidad y mortalidad, la fecundidad, la estructura poblacional y las tasas de crecimiento poblacional, y que esta distribución y abundancia impacta la estructura genética de las poblaciones, el nivel de adecuación de los individuos y, por tanto, su capacidad de adaptación al ambiente, podríamos considerar que las características demográficas de las especies son aspectos causales de la rareza, mientras que las características genéticas de las especies raras son consecuencia de los tamaños poblacionales pequeños o del aislamiento característico de las poblaciones de este tipo de especies.

El planteamiento y el análisis de argumentos como el anterior resultan trascendentales en la búsqueda e interpretación de respuestas a un sin fin de preguntas que aún nos quedan por resolver acerca de la biología de las especies vegetales raras. Por ejemplo, ¿cómo afecta la heterogeneidad ambiental al comportamiento demográfico de las especies de plantas raras?, ¿qué características demográficas y genéticas están asociadas a los diferentes tipos de rareza?, ¿qué relación existe entre los atributos demográficos, los genéticos y la restricción de hábitat?, ¿cómo afectan las diferencias a nivel filogenético y ecológico el comportamiento demográfico y genético de las especies raras?, ¿son las bajas tasas de reproducción sexual en especies vegetales raras las responsables de las bajas tasas de colonización?, ¿los patrones demográficos y genéticos descritos hasta la fecha en especies raras son causas o consecuencias de la rareza? Las respuestas a estas y otras preguntas sólo podrán resolverse en la medida en que, por un lado, se incremente el número de estudios comparativos entre especies emparentadas con diferentes niveles de rareza, que nos permita contar con análisis comparativos más reales, puesto que al comparar especies cercanas se minimiza el efecto de linaje y se puede suponer que las diferencias entre ellas son de origen reciente y posiblemente han evolucionado en respuesta a presiones específicas de selección natural. Por otro lado, es necesario que se utilicen enfoques novedosos, como aquellos en los que se integran los conocimientos generados a partir de diferentes disciplinas biológicas (i.e. demografía, genética, fisiología, 


\section{Ligia GuAdalupe EsParZa-Olguín}

interacciones bióticas, etc.). Esta falta de integración fue señalada por Schemske et al. (1994), quienes revisaron un total de 78 artículos publicados hasta esa fecha sobre la biología de especies raras; entre éstos, sólo cuatro estudios incluían información tanto genética como ecológica de manera simultánea. Diez años después todavía es poco común la aplicación de diversas disciplinas o enfoques de manera paralela en un mismo estudio, en gran parte debido a que los resultados son difíciles de compaginar. Lo anterior tiene que ver en alguna medida con el hecho de que muchos de los conceptos que utiliza la genética de poblaciones (como el efecto fundador, la deriva génica, la diferenciación genética, el flujo génico, la especiación alopátrica, el tamaño efectivo de la población, etc.) parten de la base de que las poblaciones tiene una estructura espacial, es decir, que están organizadas a manera de demes, o subpoblaciones esparcidas en el espacio (concepción que poco a poco se ha ido integrando en el concepto de metapoblación; Levins, 1969). Por otro lado, en la mayoría de los estudios que emplean un enfoque ecológico para analizar a las especies raras, no se emplea este tipo de visión, puesto que las poblaciones son concebidas como entidades "globales"; cuando se estudian varias poblaciones, éstas son consideradas como replicas o entidades separadas y no como unidades potencialmente interdependientes, es decir, como partes de una metapoblación (Silvertown, 1991). En este sentido, el surgimiento y desarrollo de la teoría metapoblacional, que ha llevado también a los demógrafos a concebir a las especies como entidades estructuradas en sub-poblaciones repartidas en el espacio, puede funcionar, como lo sugiere Silvertown (1991), como el puente que permita integrar los enfoques ecológico y genético en una nueva disciplina que seguramente podrá dar respuesta a muchas de las interrogantes que existen alrededor de la rareza. La integración de los resultados de estas dos disciplinas en el contexto de la teoría metapoblacional promete la creación de un conjunto de variables comunes que permitan analizar a las poblaciones como entidades espacialmente estructuradas, dejando atrás la concepción de la población como un sistema cerrado, la cual no dejaba de imprimirle una cierta idea de estática e irrealidad a dicho concepto.

En resumen, lo que seguramente nos llevará poco a poco a comprender el fenómeno de la rareza a mayor profundidad, serán los estudios multidisciplinarios en los cuales se analice la biología de diferentes especies congéneres con distintos niveles de rareza, abordando temas como a) sus características reproductivas (fenología, germinación, establecimiento de plántulas), b) su comportamiento demográfico (incluyendo la demografía a nivel metapoblacional), c) su estructura genética, d) sus interacciones bióticas (i.e. capacidades competitivas, asociaciones con otras especies) y e) las características de los ambientes a los que están asociadas. Esto nos permitirá tener suficiente información para empezar a entender por qué hay unas especies con una distribución más amplia y con poblaciones más abundantes que otras, que finalmente sigue siendo una de las preguntas centrales de la ecología.

\section{Agradecimientos}

Agradezco la minuciosa revisión de Teresa Valverde, Francisco Molina Freaner y Eduardo Martínez Romero, cuyos comentarios enriquecieron este manuscrito. Así mismo, agradezco los comentarios de María del Carmen Mandujano, Luis Eguiarte Fruns, Luz María Calvo y de un revisor anónimo a versiones previas de este trabajo. El presente proyecto se realizó gracias a una beca doctoral por parte de CONACyT a la autora.

\section{Literatura citada}

Allphin L., Windham M.D. y Harper K.T. 1998. Genetic diversity and gene flow in the endangered bear poppy, Arctomecon humilis (Papavaraceae). American Journal of Botany 85:12511261.

Andersen H. 1989. The rare plants of the Galápagos Islands and their conservation. Biological Conservation 47:49-77.

Arroyo M.T.K. 1975. Electrophoretic studies of genetic variation in natural populations of allogamous Limnanthes alba and autogamous Limnanthes floccosa (Limnanthaceae). Heredity 35:153-164.

Ayres D.R. y Ryan F.J. 1997. The clonal and population structure of a rare endemic plant, Wyethia reticulata (Asteraceae): allozyme and RAPD analysis. Molecular Ecology 6:761-772.

Babbel G.R. y Selander R.K. 1974. Genetic variability in edaphically restricted and widespread plant species. Evolution 28:619-630.

Baskauf C.J. y Eickmeier W.G. 1994. Comparative ecophysiology of a rare and a widespread species of Echinacea (Asteraceae). American Journal of Botany 81:958-964.

Baskin J.M., Snyder K.M., Walck J.L. y Baskin C.C. 1997. The comparative autoecology of endemic, globally-rare, and geographically-widespread, common plant species: three case studies. The Southwestern Naturalist 42:384-399.

Bayer R.J. y Crawford D.J. 1986. Allozyme divergence among five diploid species of Antennaria (Asteraceae: Inuleae) and their allopolyploid derivatives. American Journal of Botany 73:287-296.

Begon M., Harper J.L. y Townsend C.R. 1990 Ecology: Individuals, Populations and Communities. Blackwell Scientific, Oxford.

Betancourt L.J., Schuster W.S., Mitton J.B. y Anderson R.S. 1991. Fossil and genetic history of a pinyon pine (Pinus edulis) isolate. Ecology 72:1685-1696.

Bevill R.L. y Louda S.M. 1999. Comparisons of related rare and common species in the study of plant rarity. Conservation Biology 13:493-498.

Bierzychudek P. 1982. The demography of jack-in-the-pulpit, a forest perennial that changes sex. Ecological Monographs 52:335-351. 
Bijisma R., Ouborg N.J. y van Treuren R.1991. Genetic and phenotypic variation in relation to population size in two plant species: Salvia pratensis and Scabiosa columbaria. En: Seitz A. y Loeshcke V. Eds. Species Conservation: A PopulationBiological Approach, pp. 89-101, Birkhauser Verlag, Basilea.

Boyce M. 1992. Population viability analysis. Annual Review of Ecology and Systematics 23:481-506.

Byers D.L. y Meagher T.R. 1997. A comparison of demographic characteristics in a rare and a common species of Eupatorium. Ecological Applications 7:519-530.

Caswell H. 1989. Matrix Population Models. Sinauer Associates, Inc. Publishers, Sunderland, Massachusetts.

Clark-Tapia R. 2000. Estructura genética de dos cactáceas columnares del Desierto Sonorense Stenocereus gummosus y S. eruca (Cactaceae). Tesis de Maestría, Instituto de Ecología, Universidad Nacional Autónoma de México, México, D.F., $75 \mathrm{pp}$.

Clegg M.T., Horch C.R. y Church G.L. 1976. Extreme genetic similarity among northeastern species of wild rye. Genetics 83:Suppl.15.

Coates D.J. y Hamley V.L. 1999. Genetic divergence and the mating system in the endangered and geographically restricted species, Lambertia orbifolia Gardner (Proteaceae). Heredity 83:418-427.

Cody M.L. 1986. Diversity, rarity, and conservation in Mediterranean-climate regions. En: Soulé M.E. Ed. Conservation Biology: the Science of Scarcity and Diversity, pp. 122-152, Sinauer, Sunderland.

Cole C.T. y Biesboer D.D. 1992. Monomorphism, reduced gene flow, and cleistogamy in rare and common species of Lespedeza (Fabaceae). American Journal of Botany 79:567575.

Crawford D.J., Ornduff R. y Vasey M.C. 1985. Allozyme variation within and between Lasthenia minor and its derivative species L. maritime (Asteraceae). American Journal of Botany 72:1177-1184.

Cruzan M.B. 1998. Genetic markers in plant evolutionary ecology. Ecology 79:400-412.

Delgado P., Piñero D., Chaos A., Pérez-Nasser N. y ÁlvarezBuylla E.R. 1999. High population differentiation and genetic variation in the endangered Mexican pine Pinus rzedowskii (Pinaceae). American Journal of Botany 86:669-684.

Drury W.H. 1974. Rare species. Biological Conservation. 6:162169.

Edward A.L. y Sharitz R.R. 2000. Population genetics of two rare perennials in isolated wetlands: Sagittaria isoetiformis and $S$. teres (Alismataceae). American Journal of Botany 87:11471158.

Elisens W.J. y Crawford D.J. 1988. Genetic variation and diferentiation in the genus Mabrya (Scrophylariaceae-Antirrhineae). Systematic and evolutionary inferences. American Journal of Botany 75:85-96.

Esparza-Olguín L., Valverde T. y Vilchis-Anaya E. 2002. Demographic analysis of a rare columnar cactus (Neobuxbaumia macrocephala) in the Tehuacan Valley, Mexico. Biological Conservation 103:249-259.

Esselman E.J., Crawford D.J., Brauner S., Stuessy T.F., Anderson G.J. y Silva-O M. 2000. RAPD marker diversity within and divergence among species of Dendroseris (Asteraceae: Lactuceae). American Journal of Botany 87:591-596.
Evans M.E.K., Dolan R.W., Menges E.S. y Gordon D.R. 2000. Genetic diversity and reproductive biology in Warea carteri (Brassicaceae), a narrowly endemic Florida scrub annual. American Journal of Botany 87:372-381.

Falk D.A. y Holsinger K.E. 1991. Genetics and Conservation of Rare Plants. Oxford University Press, Oxford.

Feret P.P. 1974. Genetic differences among three small stands or Pinus pungens. Theoretical and Applied Genetics 44:173-177.

Fiedler P.L. 1987. Life history and population dynamics of rare and common mariposa lilies (Calochortus Pursh: Lilaceae). Journal of Ecology 75:977-995.

Fisher M. y Matthies D. 1998. RAPD variation in relation to population size and plant fitness in the rare Gentianella germanica (Gentianaceae). American Journal of Botany 85:811-819.

Franco M. 1990. Ecología de poblaciones. Ciencias Núm. Esp. 4:4-9.

Fréville H., Colas B., Ronfort J., Riba M. y Olivieri I. 1998. Predicting endemism from population structure of a widespread species: case study Centaurea maculosa Lam. (Asteraceae). Conservation Biology 12:1269-1278.

Friar E.A., Robichaux R.H. y Mount D.W. 1996. Molecular genetic variation following a population crash in the endangered Mauna Kea silversword, Argyxiphium sandwicense ssp. sandwicense (Asteraceae). Molecular Ecology 5:687-691.

García M.B. 1997. Borderea chouardii, la primera planta española que cuenta con un plan de recuperación. www.quercus.es/RedQercus/Articulos/borderea.htm

Gaston K.G. 1994. Rarity. Chapman and Hall, Londres.

Gitzendanner M.A. y Soltis P.S. 2000. Patterns of genetic variation in rare and widespread plant congeners. American Journal of Botany 87:783-792.

Godínez-Álvarez H., Valiente-Banuet A. y Valiente-Banuet L. 1999. Biotic interactions and the population dynamics of the long-lived columnar cactus Neobuxbaumia tetetzo in the Tehuacán Valley, Mexico. Canadian Journal of Botany 77:203-208.

Godt M.W., Hamrick J.L. y Bratton S. 1995. Genetic diversity in a threatened wetland species, Helonias bullata (Liliaceae). Conservation Biology 9:596-604.

Godt M.W. y Hamrick J.L. 1996. Allozyme diversity in the endangered shrub Lindera melissifolia (Asteraceae) and its widespread congener Lindera benzoin. Canadian Journal of Forest Research 26:2080-2087.

Gottlieb L.D. 1973a. Genetic differentiation, sympatric speciation, and the origin of a diploid species of Stephanomeria. American Journal of Botany 60:545-553.

Gottlieb L.D. 1973b. Enzyme differentiation and phylogeny in Clarkia franciscana, C. rubicunda and C. amoena. Evolution 27:205-214.

Gottlieb L.D. 1974. Genetic confirmation of the origin of Clardia ligulata. Evolution 28:244-250.

Gottlieb L.D. y Plitz G. 1976. Genetic similarity between Gaura longiflora and its obligately outcrossing derivate $G$. demareei. Systematic Botany 1:181-187.

Gottlieb L.D. 1977. Genotypic similarity of large and small individuals in a natural population of the annual plant Stephanomeria exigua spp. coronaria (Compositae). Journal of Ecology 65:127-134.

Gottlieb L.D., Warwick S.I. y Ford V.S. 1985. Morphological and electrophoretic divergence between Layia discoidea and $L$. 
glandulosa. Systematic Botany 10:484-495.

Griggs R.F. 1940. The ecology of rare plants. Bulletin of Torrey Botanic Club 67:575-594.

Hamrick J.L. 1983. The distribution of genetic variation within and among natural plant populations. En: Shonewald-Cox M., Chambers SM., MacBryde B. y Thomas L. Eds. Genetics and Conservation: A Reference for Managing Wild Animal and Plant Population, pp. 500-508, Benjamin Cummings, Menlo Park, California.

Hamrick J.L. y Godt M.J.W. 1989. Allozyme diversity in plant species. En: Brown A.D.H., Clegg M.T., Kahler A.L. y Weir B.S. Eds. Plant Populations Genetics, Breeding and Genetic Resources, pp. 43-63, Sinauer, Sunderland.

Hamrick J.L. y Godt M.W. 1996. Conservation genetics of endemic plant species. En: Avise J.C. y Hamrick J.L. Eds. Conservation Genetics: Case Histories from Nature, pp. 281303, Chapman and Hall, Londres.

Hamrick J.L., Linhart Y.B. y Mitton J.B. 1979. Relationships between life history characteristics and electrophoretically detectable genetic variation in plants. Annual Review of Ecology and Systematics 10:173-200.

Hamrick J.L., Nason J.D. y Fleming T.H. 2002. Genetic diversity in columnar cacti. En: Fleming T. y Valiente A. Eds. Evolution, Ecology and Conservation of columnar cacti and their mutualists. University of Arizona Press, Tucson, Arizona. pp: 122133.

Harper J.I. 1981. The means of rarity. En: Synge H. Ed. Biological Aspects of Rare Plant Conservation, pp. 189-203, John Wiley and Sons, Nueva York.

Hogbin P.M. y Peakall R. 1999. Evaluation of the contribution of genetic research to the management of the endangered plant Zieria prostrata. Conservation Biology 13:514-522.

Holsinger K.E. y Gottielb L.D. 1991. Conservation of rare and endangered plants: principles and prospects. En: Falk D.A. y Holsinger K.E. Eds. Genetics and Conservation of Rare Plants, pp. 195-208, Oxford University Press, Nueva York.

Hubbell S.P. y Foster R.B. 1986. Commonness and rarity in a Neotropical forest: implications for tropical tree conservation. En: Soulé M.E. Ed. Conservation Biology: the Science of Scarcity and Diversity, pp. 205-231, Sinauer, Sunderland.

Hunter M.L. 1996. Fundamentals of Conservation Biology. Blackwell Science, Oxford.

Karron J.D., Linhart Y.B., Chaulk C.A. y Robertson C.A. 1988. Genetic structure of populations of geographically restricted and widespread species of Astragalus (Fabaceae). American Journal of Botany 75:1114-1119.

Kennington W.J. y James S.H. 1998. Allozyme and morphometric variation in two closely related mallee species from Western Australia, Eucalyptus argutifolia and E. obtusiflora (Myrtaceae). Australian Journal of Botany 82:173-186.

Kesseli R.V. y Jain S.K. 1984. New variation and biosystematic patterns detected by allozyme and morphological comparisons in Limnanthes sect. reflexae (Limnanthaceae). Plant Systematics and Evolution 147:133-165.

Kruckerberg A.R. y Rabinowitz D. 1985 Biological aspects of endemism in higher plants. Annual Review of Ecology and Systemics 16:447-479.

Kunin W.E. y Gaston K.G. 1993. The biology of rarity: patterns, causes and consequenses. Trends in Ecology and Evolution 8:298-301.
Kunin W.E. y Gaston K.G. 1997. The Biology of Rarity: Patterns, Causes and Consequenses of Rare-Common Differences. Chapman and Hall, Londres.

Lahti T., Kemppainen E., Kurtto A. y Uotila P. 1991. Distribution and biological characteristics of threatened vascular plants in Finland. Biological Conservation 55:299-314.

Lammi A., Siikamäki P. y Mustajärvi K. 1999. Genetic diversity, population size, and fitness in central and peripheral population of a rare plant Lychnis viscaria. Conservation Biology 13:1069-1078.

Lande R. 1988. Genetics and demography in biological conservation. Science (Washington) 241:1455-1460.

Lande R. 1995. Mutation and conservation. Conservation Biology 9:782-791.

Leding F.T. y Conkle M.T. 1983. Gene diversity and genetic structure in a narrow endemic, Torrey pine (Pinus torreyana Parry ex. Carr). Evolution 37:79-85.

Les D.H. 1991. Genetic diversity in the monoecious hydrophile Ceratophyllum (Ceratophyllaceae). American Journal of Botany 78:1070-1082.

Levin D.A. 1975. Interspecific hybridization, heterozygosity and gene exchange in Phlox. Evolution 29:37-51.

Levin D.A. 1978. Genetic variation in annual Phlox: self-compatible versus self-incompatible species. Evolution 32:245-263.

Levins R. 1969. Some demographic and genetic consequences of environmental heterogeneity for biological control. Bulletin of the Entomological Society of America 15:237-240.

Levy M. y Levin D.A. 1975. Genic heterozygosity and variation in permanent translocation heterozygotes of the Oenothera biennis complex. Genetics 79:493-512.

Lewis P.O. y Crawford D.J. 1995. Pleistocene refugium endemics exhibit greater allozymic diversity than widespread congeners in the genus Polygonella (Polygonaceae). American Journal of Botany 82:141-149.

Linhart Y.B. y Premoli A.C. 1993. Genetic variation in Aletes acaulis and its relative, the narrow endemic A. humilis (Apiaceae). American Journal of Botany 80:598-605.

Louda S.M. 1994. Experimental evidence for insect impact on populations of short-lived, perennial plants, and its application in restoration ecology. En: Bowles M.L. y Whelan C.J. Eds. Restoration of Endangered Species, pp. 118-138, Cambridge University Press, Cambridge.

Loveless MD. y Hamrick J.L. 1984. Ecological determinants of genetic structure in plant populations. Annual Review of Ecology and Systematics 15:65-95.

Loveless M.D. y Hamrick J.L. 1988. Genetic organization and evolutionary history in two North American species of Cirsium. Evolution 42: 254-265.

Lynch A.J.J. y Vaillancourt R.E. 1995. Genetic diversity in the endangered Phebalium daviessii (Rutaceae) compared to that in two widespread congeners. Australian Journal of Botany 43:181-191.

Maki M. y Horie S. 1999. Random amplified polymorphic DNA (RAPD) markers reveal less genetic variation in the endangered plant Cerastium fischerianum var. molle than in the widespread conspecific C. fischerianum var. fischerianum (Caryophyllaceae). Molecular Ecology 8:145-150.

Martínez-Palacios A., Eguiarte L.E. y Furnier G.R. 1999. Genetic diversity of the endangered endemic Agave victoriae-reginae (Agavaceae) in the Chihuahuan Desert. American Journal of 
Botany 86:1093-1106.

McLeod M.J., Guttman S.I., Eshbaugh W.H. y Rayle R.E. 1983. An electrophoretic study on evolution in Capsicum (Solanaceae). Evolution 37:562-574.

Menges E. 1990. Population viability analysis for an endangered plant. Conservation Biology 4:52-62.

Milligan B.G., Leebens-Mack J. y Strand A.E. 1994. Conservation genetics beyond the maintenance of marker diversity. Molecular Ecology 3:423-435.

Moran G.F., Bell J.C. y Matheson A.C. 1980. The genetic structure and levels of inbreeding in a Pinus radiata D. Don seed orchard. Silvae Genetica 29:190-193.

Moran G.F. y Hopper S.D. 1983. Genetic diversity and the insular population structure of the rare granite rocks species Eucalyptus caesia Benth. Australian Journal of Botany 31:161172.

Navarro-Quezada A.R. 1999. Estructura genética y procesos de especiación de Agave cerulata (Trel.) y Agave subsimplex (Trel.) en el desierto Sonorense a partir de RAPD's. Tesis de Licenciatura, Facultad de Ciencias, Universidad Nacional Autónoma de México, México D.F., 82 pp.

Niebling A.V. y Conkle M.T. 1990. Diversity of washoe pine and comparisons with allozymes of ponderosa pine races. Canadian Journal of Forest Research 20:298-308.

O'Brien S.J. 1994. The cheetah's conservation controversy. Conservation Biology 8:1153-1155.

Oostermeijer J.G.B., van Eijck M.W. y den Nijs J.C.M. 1994. Offspring fitness in relation to population size and genetic variation in the rare perennial plant species Gentiana pneumonanthe (Gentianeceae). Oecologia 97:289-296.

Ouborg N.J. y van Treuren R. 1995. Variation in fitness-related characters among small and large population of Salvia pratensis. Journal of Ecology 83:369-380.

Pate J.S. y Hopper S.D. 1994. Rare and common plants in ecosystems, with special reference to the South-west Australian flora. En: Schulze E.D. y Mooney H.A. Eds. Biodiversity and Ecosystems Function, pp. 293-325, Springer Verlag, Berlín.

Pleasants J.M. y Wendel J.F. 1989. Genetic diversity in a clonal narrow endemic, Erythronium propullans, and in its widespread progenitor, Erythronium albidum. American Journal of Botany 76:1136-1151.

Primack R.B. 1993. Essentials of Conservation Biology. Sinauer Associates, Massachusetts.

Prober S.M. 1992. Environmental influences on the distribution of the rare Eucalyptus paliformis and the common E. fraxinoides. Australian Journal of Ecology 17:51-65.

Prober S.M. y Austin M.P. 1990. Habitat peculiarity as a cause of rarity in Eucaliptus paliformis. Australian Journal of Ecology 16:189-205.

Purdy B.G. y Bayer R.J. 1995a. Allozyme variation in the Athabasca sand dune endemic, Salix silicicola, and the closely related widespread species, S. alaxensis. Systematic Botany 20:179-190.

Purdy B.G. y Bayer R.J. 1995b. Genetic diversity in the tetraploid sand dune endemic Deschampsia mackenzieana and its widespread diploid progenitor D. cespitosa (Poaceae). American Journal of Botany 82:121-130.

Purdy BG. y Bayer R.J. 1996. Genetic variation in populations of the endemic Achillea millefolim ssp. megacephala from the Athabasca sand dunes and the widespread ssp. lanulosa in western North America. Canadian Journal of Botany 74:1138-1146.

Rabinowitz D. 1981. Seven forms of rarity. En: Synge H. Ed. Biological Aspects of Rare Plant Conservation, pp. 205-217. John Wiley y Sons, Nueva York.

Rabinowitz D., Cairns S. y Dillon T. 1986. Seven forms of rarity and their frecuency in the flora of the British Isles. En: Soulé M.E. Ed. Conservation Biology: the Science of Scarcity and Diversity, pp. 182-203, Sinauer, Sunderland.

Rabinowitz D., Rapp J.K., Cairns S. y Mayer M. 1989. The persistence of rare prairie grasses in Missouri: environmental variation buffered by reproductive output of sparse species. The American Naturalist 136:328-344.

Ranker T.A. 1994. Evolution of high genetic variability in the rare Hawaiian fern Adenophorus periens and implications for conservation management. Biological Conservation 70:19-24.

Rick C.M., Kesichi E., Fobes J.F. y Holle M. 1976. Genetic and biosystematic studies on two new sibling species of Lycopersicon from interandean Peru. Theoretical and Applied Genetics 47:55-68.

Rick C.M., Fobes J.F. y Holle M. 1977. Genetic variation in Lycopersicon pimpinellifolium: evidence of evolutionary change in mating systems. Plant Systematic Evolution 127:139-170.

Roose M.L. y Gottlieb L.D. 1976. Genetic and biochemical consequences of polyploidy in Tragopogon. Evolution 30:818-830.

Root K.V. 1998. Evaluating the effects of habitat quality, connectivity, and catastrophes on a threatened species. Ecological Applications 8:854-865.

Rossetto M., Weaver P.K. y Dixon K.W. 1995. Use of RAPD analysis in devising conservation strategies for the rare and endangered Grevillea scapigera (Proteaceae). Molecular Ecology 4:321-329.

Scogin R. 1973. Leucine aminopeptidase polymorphism in the genus Lupinus (Leguminoseae). Botanical Gazette 134:73-76.

Shapcott A. y Playford J. 1996. Comparison of genetic variability in remnant and wide-spread rain forest understory species of Austromyrtus (Myrtaceae). Biodiversity and Conservation 5:881-895.

Schemske D.W., Husband B.C., Ruckelshaus M.H., Goodwillie I., Parker M, y Bishop J.G. 1994. Evaluating approach to the conservation of rare and endangered plants. Ecology 75:584-606.

Schmazel R.J., Reichenbacher F.W. y Rutman S. 1995. Demography study of the rare Coryphantha robbinsorum (Cactaceae) in Southeastern Arizona. Madroño 42:332-348.

Sherman-Broyles S.L., Gibson J.P., Hamrick J.L., Bucher M.A. y Gibson M.J. 1992. Comparison of allozyme diversity among rare and widespread Rhus species. Systematic Botany 17:551559.

Silvertown J. 1991. Dorothy's dilema and the unification of plant population biology. Trends in Ecology and Evolution 6:346348.

Smith J.F. y Pham T.V. 1996. Genetic diversity of the narrow endemic Allium aaseae (Alliaceae). American Journal of Botany 83:717-726.

Soltis P.S., Soltis, D.E, Tucker T.L. y Lang F.A. 1992. Allozyme variability is absent in the narrow endemic Bensoniella oregona (Salfragaceae). Conservation Biology 6:131-134.

Soltis P.S., Soltis D.E. y Norvell T.L. 1997. Genetic diversity in rare and widespread species of Lomatium (Apiaceae). Madroño 


\section{Ligia GuAdALUPE EsParZA-Olguín}

44:59-73.

Soulé M.E. 1986. Patterns of diversity and rarity: their implications for conservation. En: Soulé M.E. Ed. Conservation Biology: the Science of Scarcity and Diversity, pp. 111-124, Sinauer Associates, Sunderland.

Stearns S. 1992. The Evolution of Life Histories. Oxford University Press, Oxford.

Stebbins G.L. 1980. Rarity of plant species: a synthetic viewpoint. Rhodora 82:77-86.

Tansley S.A. y Brown C.R. 2000. RAPD variation in the rare and endangered Leucadendron elimense (Proteaceae): implications for their conservation. Biological Conservation 95:39-48.

van Groenendael J., Kroon H.D. y Caswell C.H. 1988. Projection matrices in population biology. Trends in Ecology and Evolution 3:264-269.

Vogelmann J.E. y Gastony G.J. 1987. Electrophoretic enzyme analysis of North American and Eastern Asian populations of Agastache sect. Agastache (Labiatae). American Journal of Botany 74:385-393.

Walck J.L., Baskin J.M. y Baskin C.C. 1999. Relative competitive abilities and growth characteristics of a narrowly endemic and a geographically widespread Solidago species (Asteraceae). American Journal of Botany 86:820.

Waller D.M., O'Malley D.M. y Gawler S.C. 1987. Genetic variation in the extreme endemic Pedicularis furbishiae (Scrophulariaceae). Conservation Biology 1:335-340.

Warwick S.I. y Gottlieb L.D. 1985. Genetic divergence and geographic speciation in Layia (Compositae). Evolution 39:12361241.

Wong K.C. y Sun M. 1999. Reproductive biology and conservation genetics of Goodyera procera (Orchidaceae). American Journal of Botany 86:1406-1416.

Woolley A. y Kirkpatrick J.B. 1999. Factors related to condition and rare and threatened species occurrence in lowland, humid basalt remnants in northern Tasmania. Biological Conservation 87:131-142.

Young A.B. y Brown H.D. 1996. Comparative population genetic structure of the rare woodland shrub Daviesia suaveolens and its common congener D. mimosoides. Conservation Biology 10:1220-1228.

Fecha de recepción: 9 de abril de 2003

Versión corregida: 26 de julio de 2004

Aceptado: 3 de septiembre de 2004 